\title{
Differential Antagonistic Games with Lexicographic Vector-Payoffs
}

\author{
Guram N. Beltadze \\ Departament Artifical Intelligence Georgian Technical University, Georgia, \\ Tbilisi, 0175, str. Kostava 77 \\ Email: gbeltadze@yahoo.com
}

Received: 30 December 2018; Accepted: 20 January 2019; Published: 08 March 2019

\begin{abstract}
In this paper the existence problem of the equilibrium situation in differential antagonistic games with perfect information and lexicographic payoffs or in a $m$-dimensional vector-payoffs' game where criteria are strictly ranged with preference relation is studied. The players' dinamic is defined by vector differential $\dot{x}=f(t, x, u), \quad \dot{y}=g(t, y, v)$ equations, respectively control functions $u(\cdot), v(\cdot)$ and time $t \in[0, T]$ interval. This is a game $\Gamma^{L}\left(x_{0}, y_{0}\right) \equiv\left(\Gamma^{1}, \ldots, \Gamma^{m}\right)$ where $x_{0}, y_{0}$ are starting positions in $t=0$ moment respectively the first and second players'. $x(t)$ and $y(t)$ are trajectories, the players final aim is finding their optimal variants. A lexicographic $\varepsilon$-equilibrium situation is defined in the game and the conditions of its existence are investiga-terd These conditions are mainly about $f$ and $g$ functi-ons. The main definitions are introduced and some results are formulated from theory of differential games with scalar payoff functions and independent move-ments, they are the main for getting results for analogic differential games in the case of lexicographic payoffs. Some auxiliary statements correctness are also establi-shed, on its basic it is proved that in $\Gamma^{L}\left(x_{0}, y_{0}\right)$ game for any $\varepsilon>0$ there exists a lexicographic $\varepsilon$-equilibrium situation in pure strategies.
\end{abstract}

Index Terms-Lexicographic, Antagonistic, Differential game, Equilibrium, $\varepsilon$-equilibrium.

\section{INTRODUCTION}

In one of the new areas of optimal control theory - the theory of differential games, problems of the pursuit of one controlled object by another are studied; in these problems features appear pertaining to the attainability of different, conflicting objectives. In problems of pur-suit the partners tend to select strategies in order that the chosen criteria give different values, they are in conflict. Another, more general problems in differen-tial games, the partners have their own indices of quality that are different, but depend upon the strategy of each player; the players tend to choose strategies that are best in the sense of their own indices of quality [1].

The peculiarity of differential games rests in the fact that for optimization of each index of quality there is a characteristic control resource. In control theory the situation is different. The rule of consumption of cont-rol resource is chosen so that all performance indices of the system take on their best values. However, the selection of a control law is effected by optimizing only one of the chosen criteria, which as noted above, is not sufficient in many practical situations.

The lack of control rules that are well defined through conditions of optimizing a scalar criterion leads to the following: The selection and optimization of a given functional answers to only of the require-ments of the control system, while other requirements, that are often equally important, are ignored. The tota-lity of all requirements may be accounted for by a collection of functionals forming a vector of criterion functions, i.e., a vector-valued criterion. Very naturally there arise mathematical problems requiring the simultaneous optimization of a collection of functionals, mesch of which measures a definite aspect of the sys-tem. Important problems of simultaneous optimization of two or more functionals are repeatedly noted by Soviet and foreign scholars $[2,3]$.

The paper [4] is devoted to the investigation and solution of differential games with a vector-valued payoff function. Slater, Pareto and Geoffrion - optimal strategies are defined for such a game, and their positive and negative propertes are examined. A new class of solutions called vector-valued guarantees is proposed and their properties examined. According to the authors, this new approach is superior primarily because it has the properties of equivalence and interchangeability - antagonistic players should be able to achieve guaranteed re-sults simultaneously. The approach is tested in a com-petition problem, as well as a pursuit game with noise.

In the given paper we discuss studying antagonistic differential game with $m$-dimensional vector-payoffs, their criterion are strictly ranged. That means that we'll define differential antagonistic game with lexicographic payoffs. We'll study the problem of the existence Nash equilibrium in it with usual standard conceptions. 
Let define $m$-dimensional antagonistic lexicographic strategic game with Nash equilibrium.

Let's note $X$ and $Y$ are the sets of the first and the second players' accordingly in antagonistic game $\Gamma=<N=\{1,2\}, X, Y, H>$, but the first player's payoff, $H$ is $m$-dimensional a vector-function $H=\left(H^{1}, H^{2}, \ldots, H^{m}\right)$ and on the set of situations $X \times Y$ their comparison holds lexicographically. We call such game a lexicog-raphic antagonistic game with $m$-dimensional and we note it in the following way

$$
\begin{aligned}
\Gamma^{L}=<N= & \{1,2\}, X, Y, H=\left(H^{1}, \ldots, H^{m}\right)> \\
& \equiv\left(\Gamma^{1}, \ldots, \Gamma^{m}\right)
\end{aligned}
$$

For two $a=\left(a_{1}, \ldots, a_{m}\right)$ and $b=\left(b_{1}, \ldots, b_{m}\right) \quad$ vectors lexicographic preference $a \succ^{L} b$ means that it fulfills one of the following $m$ conditions:

$$
\text { 1) } \begin{aligned}
a_{1} & \left.\left.>b_{1} ; 2\right) a_{1}=b_{1}, a_{2}>b_{2} ; \ldots ; m\right) \\
a_{1} & =b_{1}, \ldots, a_{m-1}=b_{m-1}, a_{m}>b_{m}
\end{aligned}
$$

and $a \succcurlyeq^{L} b$ if $a \succ^{L} b$ or $a=b$.

By means of Game Theory the main exclusivity of modelling of strategic conflict is finding of J. Nash equilibrium (steady) situation on the basis of analysis of the model of corresponding game. Consequently, in $\Gamma^{L}$ game the main principle of optimality is Nash equilibrium situation. For its determination let's indicate the situation in the given game $(x, y) \in X \times Y$.

Definition 1.1. The situation $\left(x^{*}, y^{*}\right) \in X \times Y$ is called Nash equilibrium situation (or saddle point) in $\Gamma^{L}$ game, if for $\forall x \in X$ and $\forall y \in Y$ for the strategies will fulfill the inequalities:

$$
H\left(x, y^{*}\right) \preccurlyeq^{\mathrm{L}} H\left(x^{*}, y^{*}\right) \preccurlyeq^{\mathrm{L}} H\left(x^{*}, y\right) .
$$

Let's suppose that $\Gamma^{L}$ is $n$ player's $N=\{1, \ldots, n\}$ lexicographic strategic game and the set of equilibrium situation of its standard mixed extension note by $\sigma\left(\Gamma^{L}\right)$. In such kind of games the main problem is that in every $\Gamma^{L}$ game there doesn't exist the equilibrium situation or maybe this set will be empty $-\sigma\left(\Gamma^{L}\right)=\varnothing$ [5-14].

Lexicographic antagonistic defferential games as lexicographic stochastic games [15], belong to the class of dynamic games.

We'll discuss such games with the given duration and independent movements. Suppose that the first and the second players' condition in every $t$ moment of the duration of the play are described accordingly by relevant of euclidean $R^{n_{1}}$ and $R^{n_{2}}$ spaces.

For the solution of scalar differential games by different authors are constructed different approaches by using two types of solutions - saddle point and $\varepsilon$-saddle points existence was proved. For example in [16] paper the existence of saddle point was proved, but it is rather big size and from the intuitive point of view it is difficult to get. But the existence of $\varepsilon$-saddle point was proved rather easily for example in [17-22] papers.

In the process of analysis of the above mentioned approaches it is obvious that the easiest and direct method of the construction of lexicographic differential games is possible on the basis of the second type for-mulization. This kind of approach gives us the oppor-tunity to prove lexicographic $\varepsilon$-equilibrium in lexicog-raphic differential games by using a simple mathema-tical apparatus.

\section{THE MAIN DEFINITIONS AND SUBSIDIARY RESUltS}

In our provement we'll use generalized lemma on the epimorphism in lexicographic case, that was not used by the above mentioned authors for scalar games.

In order to formulate lemma for the players $N=\{1, \ldots, n\}$ set let's discuss in $\alpha$ situation payoff vectoor-functions $H_{i}(\alpha)=\left(H_{i}^{1}(\alpha), \ldots, H_{i}^{m}(\alpha)\right)$ and matrix

$$
H(\alpha)=\left(\begin{array}{lll}
\left(H_{1}^{1}(\alpha),\right. & \ldots & \left., H_{1}^{m}(\alpha)\right) \\
\cdot & \cdots & \cdot \\
\left(H_{n}^{1}(\alpha),\right. & \cdots & \left., H_{n}^{m}(\alpha)\right)
\end{array}\right)
$$

Get some norms

$$
\begin{aligned}
& \left\|H_{i}(\alpha)\right\|=\max _{1 \leq k \leq m}\left|H_{i}^{k}(\alpha)\right|, \\
& \|H(\alpha)\|=\max _{1 \leq i \leq n, 1 \leq k \leq m}\left|H_{i}^{k}(\alpha)\right| .
\end{aligned}
$$

Let's define $n$ player's finite $m$-dimensional lexicographic strategic game and $\varepsilon$-equilibrium in it [22]. Suppose

$$
\Gamma^{L}=<N,\left\{S_{i}\right\}_{i \in N},\left\{H_{i}\right\}_{i \in N}>\equiv\left(\Gamma^{1}, \ldots, \Gamma^{m}\right)
$$

is such kind of game.

Let's take the smallest positive numbers $\varepsilon^{1}>0$, $\ldots, \varepsilon^{m}>0$ and consider the vector $(\varepsilon)=\left(\varepsilon^{1}, \ldots, \varepsilon^{m}\right)$.

Definition 2.1. The situation $s^{*}=\left(s_{1}{ }^{*}, \ldots, s_{n}^{*}\right)$ is called an lexicographic $\varepsilon$-equilibrium situation in game $\Gamma^{L}=\left(\Gamma^{1}, \ldots, \Gamma^{m}\right)$ according to Nash, if for any player $i=1, \ldots, n$ the following condition is fulfilled:

$$
H_{i}\left(s_{i}^{*}, s_{-i}^{*}\right) \succcurlyeq{ }^{L} H_{i}\left(s_{i}, s_{-i}^{*}\right)-(\varepsilon), \forall s_{i} \in S,
$$


where

$$
\left(s_{i}^{*}, s_{-i}^{*}\right)=s^{*}\left(s_{i}, s_{-i}^{*}\right)=\left(s_{1}^{*}, \ldots, s_{i-1}^{*}, s_{i}, s_{i+1}^{*}, \ldots, s_{n}^{*}\right) .
$$

Lemma 2.1. Suppose lexicographic strategic games

$$
\Gamma_{1}^{L}=<N,\left\{S_{i}^{\prime}\right\}_{i \in N},\left\{H_{i}^{\prime}\right\}_{i \in N}>, \Gamma_{2}^{L}=<N,\left\{S_{i}\right\}_{i \in N},\left\{H_{i}\right\}_{i \in N}>
$$

are connected to each other by means of epimorphism mappings $\mu_{i}: S_{i} \rightarrow S_{i}^{\prime}, i=1, \ldots, n$. At the same time in the situations for such epimorphisms $s=\left(s_{1}, \ldots, s_{n}\right)$ and $\mu(s)=\left(\mu_{1}(s), \ldots, \mu_{n}(s)\right)$ the following inequation $\left\|H(s)-H^{\prime}(\mu(s))\right\| \leq \varepsilon$ is correct. If $s$ is a lexicographic $\varepsilon$-equilibrium in $\Gamma_{2}^{L}$ game, then $\mu(s)$ will be lexicographic $3 \varepsilon$-equilibrium $\Gamma_{1}^{L}$ game.

Let's get the main definitions an formulate some results from the theory of differential games with scalar payoff functions and independent movements, they are main for getting results for analogic differential games in the case of lexicographic payoffs.

Suppose the first player's dynamic is defined by vector differential equation

$$
\dot{x}=f(t, x, u)
$$

but the second player's dynamic is defined by vector differential equation

$$
\dot{y}=g(t, y, v) \text {. }
$$

In the given equations $u(\cdot)$ and $v(\cdot)$ respectively the first and the second players' controlling functions, they are satisfied the limitations $u(\cdot) \in U, v(\cdot) \in V$, where $U \subset R^{p}, V \subset R^{q}$ - fixed compact sets; $t \in[0, T]$ defines time; $x(t) \in R^{n_{1}}$ and $y(t) \in R^{n_{2}}$ are phase states respectively for the first and the second players' in $t$ moment of the time.

The play begins in $t=0$ moment and its process lasts till $t=T<\infty$ moment that is defined beforehand, the fist player from the second player gets lexicographic vectorpayoff $H(x, y)=\left(H^{1}(x, y), \ldots, H^{m}(x, y)\right)$, where $x(\cdot), y(\cdot)$ are trajectories respectively for the first and second players'.

Suppose $x_{0}, y_{0}$ are starting positions in $t=0$ moment respectively for the first and second players'. The first player tries lexicographic maximization of $H(x, y)$ function, but the second player its lexicographic minimization. In such situation we have antagonistic lexicographic differential game with vector-payoff $H=\left(H^{1}, H^{2}, \ldots, H^{m}\right)$ and note this game by $\Gamma^{L}\left(x_{0}, y_{0}\right)$.

Let's formulate the conditions that must be satisfied the first player's dynamic defined differential equations system (such kind of conditions are formulated analogically for the second player):

1) For every $t \in[0, T]$ function $f$ is continuous by $(x, u) \in R^{n_{1}} \times U$, measurable by $(x, u) \in R^{n_{1}} \times U$ for every $(x, u)$;

2) There exists such measurable and integrable function on finite intervals $K$, that for any $u \in U, x, x^{\prime} \in R^{n_{1}}$ the inequation is fulfilled

$$
\left|f(t, x, u)-f\left(t, x^{\prime}, u\right)\right|_{n_{1}} \leq K(t)\left|x-x^{\prime}\right|_{n_{1}},
$$

where $|\cdot|_{n_{1}}$ note euclidean norm in space by $R^{n_{1}}$;

3) There exists such positive numbers $M, N$ and measurable function $l$, that is at the same time integrable on finite intervals, that means for every $x \in R^{n_{1}}$, $u \in U$ points continuous conditions for the solutions will be fulfilled

$$
|f(t, x, u)|_{n_{1}} \leq l(t)\left(M+N|x|_{n_{1}}\right.
$$

4) Condition vectors convexity: for every $x \in R^{n_{1}}$, $t \in[0, T]$ the set

$$
f(t, x, U)=\{f(t, x, u) \mid u \in U\}
$$

is convex.

Definition 2.1. 1. Measurable $u$ function is called the first player's admissible control on $[0, T]$ interval of the play, if each $t \in[0, T]$ the condition $u(t) \in U$ will be fulfilled.

2. The solution of (8) system is called admisible trajectory on $t \in[0, T]$ interval by starting in $x(0)=x_{0}$ point and finishing in $x(T)$ point, if it is formulated from the system of admissible control.

Analogically will be defined admissible control and admissible trajectory for the second player.

Differential game's description is comparatively easy, when the game has perfect information where in the process of choosing the meanings of the control $u(t)$ and $v(t)$ functions at every $t$ moment, phase $x(t)$ and $y(t)$ states are known. Sometimes, it is demanded that one of the player, for example the first player at every moment $t$ should know $v(t)$, that chooses the second player at this moment. In this case the second player is discriminative, but the game itself is called the game with the second player's discrimination. At this kind of definition of the perfect information the analog with perfect information for multistep (positive) games are considered. 
Let's note by $X\left(x_{0}, T\right)$ the first player's every admisable set of $x$ trajectories that is defined in $[0, T]$ interval by starting in $x_{0}$ point and by finishing in $x(T)$ point. Analogically, the second player's every admissible $y$ set of trajectories corresponding with parameters note by $Y\left(y_{0}, T\right)$.

Let's discuss $X\left(x_{0}, T\right)$ set as a subset of $C_{T}^{n_{1}}$ Banach space, that is in $[0, T]$ interval $R^{n_{1}}$ space the continuous mappings' space norm $\|x\|_{n_{1}}=\max _{t \in[0, T]}|x(t)|_{n_{1}}$, where $|\cdot|_{n_{1}}$ is $R^{n_{1}}$ space norm. Analogically note by $C_{T}^{n_{2}}$ Banach space that consists $Y\left(y_{0}, T\right)$ of the second player's trajectories.

Below we need the result consists of two parts from control theory [17,21]. Its first part is from $f(t, x, U)$ and $g(t, y, V)$ sets convexity, but the second part above mentioned 2) conditions.

Theorem 2.1. 1. Suppose $X_{0} \subset R^{n_{1}}, Y_{0} \subset R^{n_{2}}$ are compact sets, then trajectories sets

$$
\bigcup_{x_{0} \in X_{0}} X\left(x_{0}, T\right) \subset C_{T}^{n_{1}}, \bigcup_{y_{0} \in Y_{0}} Y\left(y_{0}, T\right) \subset C_{T}^{n_{2}}
$$

are compact with corresponding measure;

2. Compact sets $X(\cdot, T), Y(\cdot, T)$ with continuous form depend on their arguments in Hausdorff's measure.

Let's note

$$
X_{T}=\bigcup_{x_{0} \in X_{0}} X\left(x_{0}, T\right), Y_{T}=\bigcup_{y_{0} \in Y_{0}} Y\left(y_{0}, T\right) .
$$

for compact $X_{0} \subset R^{n_{1}}, Y_{0} \subset R^{n_{2}}$ sets.

Let's take also next result, that gives estimation among trajectories, that is formed by some control and trajectory, it is given from this control by delay of little $\delta$ time [18].

Theorem 2.2. For every $\delta \in(0,1)$ there exists the first player's trajectories mapping in itself $\Pi_{\delta}^{X}: X_{T} \rightarrow X_{T}$, that has the following features:

1. If $x \in X\left(x_{0}, T\right)$, then $\Pi_{\delta}^{X}(x) \in X\left(x_{0}, T\right)$;

2. If two trajectory $x, x^{\prime} \in X_{T}$ satisfies the conditions $x(\tau)=x^{\prime}(\tau), \quad \tau \in(0, t), \quad$ then relation $\Pi_{\delta}^{X}(x(\tau))=\Pi_{\delta}^{X}\left(x^{\prime}(\tau)\right), \tau \in[0, t+\delta T]$ is correct.

3. Suppose

$$
\varepsilon(\delta)=\sup _{x \in X_{T}}\left\{\left\|x-\prod_{\delta}^{X}(x)\right\|_{n_{1}}\right\} .
$$

Then $\lim _{\delta \rightarrow 0} \varepsilon(\delta)=0$.

\section{THE EXISTENCE OF LEXICOGRAPHIC $\varepsilon$ - EQUILIBRIUM IN LEXICOGRAPHIC DIFFERENTIAL GAMES}

Let's take the new type strategies in discrete multistage games, that we'll use in basic continuous $\Gamma^{L}\left(x_{0}, y_{0}\right)$ game.

For any intege $n \geq 0$ note $\delta=\frac{1}{2^{n}}$ and note $A_{\delta}\left(x_{0}, y_{0}\right)$ every mapping set (the first player's strategies) $\alpha_{\delta}: Y\left(y_{0}, T\right) \rightarrow X\left(x_{0}, T\right)$, such kind of that, if $y, y^{\prime} \in Y\left(y_{0}, T\right) \quad$ trajectories fulfill equality $y(\tau)=y^{\prime}(\tau), \quad \tau \in[0, i \delta T], \quad$ then for $\tau \in[0,(i+1) \delta T]$, $i=0,1, \ldots, \frac{1}{\delta}-1$ numbers the following equality will be fulfilled $\alpha_{\delta}(y(\tau))=\alpha_{\delta}\left(y^{\prime}(\tau)\right)$.

Now note by $A^{\delta}\left(x_{0}, y_{0}\right)$ every set of mapping (they are again the first player's strategies) $\alpha^{\delta}: Y\left(y_{0}, T\right) \rightarrow X\left(x_{0}, T\right)$, such as if $y, y^{\prime} \in Y\left(y_{0}, T\right)$ trajectories fulfills the equality $\quad y(\tau)=y^{\prime}(\tau), \quad \tau \in[0, i \delta T], \quad$ then for $\tau \in[0,(i+1) \delta T]$, numbers the following equality $\alpha^{\delta}(y(\tau))=\alpha^{\delta}\left(y^{\prime}(\tau)\right)$ will be fulfilled.

Sets of strategies for the second player $B_{\delta}\left(x_{0}, y_{0}\right), B^{\delta}\left(x_{0}, y_{0}\right), \beta_{\delta}, \beta^{\delta} \quad$ will be defined analogically.

We'll discuss sets of $A_{\delta}$ and $A^{\delta}$ strategies as $Y\left(y_{0}, T\right)$ set in $X\left(x_{0}, T\right)$ set every mapping topolo-gical $F\left(Y\left(y_{0}, T\right), X\left(x_{0}, T\right)\right)$ space set of a space by pointlike topology of convergence. Analogically will be discussed the set of a second player's strategies $B_{\delta}$ and $B^{\delta}$, as the sets of $F\left(X\left(x_{0}, T\right), Y\left(y_{0}, T\right)\right)$ space.

Theorem 3.1. For every $\delta_{1} \leq \delta_{2}$ numbers the following inclusions are correct $A_{\delta_{2}} \subset A_{\delta_{1}} \subset A^{\delta_{1}} \subset A^{\delta_{2}}$, $B_{\delta_{2}} \subset B_{\delta_{1}} \subset B^{\delta_{1}} \subset B^{\delta_{2}}$.

The sets $A_{\delta}(\cdot), A^{\delta}(\cdot)$ closed and that is why they are compactives, as the subsets of $F\left(Y\left(y_{0}, T\right), X\left(x_{0}, T\right)\right)$ space. Analogically, $B_{\delta}$ and $B^{\delta}$ sets are closed and that is why, as the subsets of $F\left(X\left(x_{0}, T\right), Y\left(y_{0}, T\right)\right)$ space.

Proof. The first part of the theorem comes from the definitions, but the proof of the second part we can find in [19].

Above defined a $\alpha^{\delta}, \beta^{\delta}$ strategies are such strate-gies that they give the players opportunities that by turn in discrete games at every stage choosing control after 
choosing control by one player, or for the second time, as a result these strategies make discrimination of the opposite side. Using $\Pi_{\delta}^{X}$ mapping that is delay mapping, takes away their opportunites and the play becomes a discriminating one. e.t.c. $\Pi_{\delta}^{X}$ mapping contracts the player's informative opportunities and transform the strategy into $A_{\delta}$ (or $B_{\delta}$ ) class. Therefore from the theorem 2.2 follows the following theorem's correctness.

Theorem 3.2. Suppose $\alpha^{\delta} \in A^{\delta}, \beta^{\delta} \in B^{\delta}$, then functions' composition following belongings are correct

$$
\Pi_{\delta}^{X} \circ \alpha^{\delta} \in A_{\delta}(\cdot), \beta^{\delta} \circ \Pi_{\delta}^{X} \in B_{\delta}(\cdot)
$$

For every $\alpha^{\delta} \in A^{\delta}, y \in Y\left(y_{0}, T\right)$ the following ine-quation is correct

$$
\left|\alpha^{\delta}(y)-\left(\Pi_{\delta}^{X} \circ \alpha^{\delta}\right)(y)\right| \mid \leq \varepsilon(\delta)
$$

where $\varepsilon(\delta) \rightarrow 0$ when $\delta \rightarrow 0$.

For antagonistic lexicographic differential $\Gamma^{L}\left(x_{0}, y_{0}\right)$ game let's discuss auxiliary discrete games with per-fect information and the players discriminatios, we note that for the first and second player respectively $\Gamma^{L, \delta}\left(x_{0}, y_{0}\right)$ and $\Gamma_{\delta}^{L}\left(x_{0}, y_{0}\right)$. By $\Gamma^{L, \delta}(\cdot)$ let's call the game majority or upper game, but $\Gamma_{\delta}^{L}(\cdot)$ game - minorized or lower game.

In upper $\Gamma^{L, \delta}(\cdot)$ gamer the second player chooses $\beta^{\delta}$ strategy, but the first player $-\alpha^{\delta}$ strategy. The only couple of trajectories $(x, y) \in X\left(x_{0}, T\right) \times Y\left(y_{0}, T\right)$ and the situation $\left(\alpha^{\delta}, \beta_{\delta}\right)$ are defined on $[0, \delta T],[\delta T, 2 \delta T]$, $\ldots,[T-\delta T, T]$ intervals in the following way.

By means of definition of $\beta_{\delta}$ strategy, for every $x_{1}, x_{2} \in X\left(x_{0}, T\right)$ we have the equality $t \in[0, \delta T]$. In this situation let's note by $y(t)=\beta_{\delta}\left(x_{1}(t)\right), t \in[0, \delta T]$ for every $x_{1} \in X\left(x_{0}, T\right)$.

If $y_{1}, y_{2} \in Y\left(y_{0}, T\right)$ are any type trajectories, such as, that for $t \in[0, \delta T] \quad y_{1}(t)=y_{2}(t)=y(t)$, then by the definition of $\alpha^{\delta}$ strategy we have equality $\alpha^{\delta}\left(y_{1}(t)\right)=\alpha^{\delta}\left(x_{2}(t)\right), t \in[0, \delta T]$. In this situation let's note

$$
x(t)=\alpha^{\delta}\left(y_{1}(t)\right), t \in[0, \delta T]
$$

where $y_{1} \in Y\left(y_{0}, T\right)$ the second player's any type of trajectory such as that $y_{1}(t)=y(t), t \in[0, \delta T]$.
If $x_{1}, x_{2} \in X\left(x_{0}, T\right)$ are trajectories of the first player's, that for $t \in[0, \delta T]$ fulfills the equality $x_{1}(t)=x_{2}(t)=x(t)$, then by the definition of $\beta_{\delta}$ strategy we will have the equality $\beta_{\delta}\left(x_{1}(t)\right)=\beta_{\delta}\left(x_{2}(t)\right), t \in[0,2 \delta T]$. In this situation lets note

$$
y(t)=\beta_{\delta}\left(x_{1}(t)\right), t \in[0,2 \delta T]
$$

where $x_{1} \in X\left(x_{0}, T\right)$ is the first player's such trajec-tory, for which fulfills the equality $x_{1}(t)=x(t), t \in[0, \delta T]$.

Analogically to the second step the second player's $y$ strategy's knowledge on $[0,2 \delta T]$ interval by means of $\alpha^{\delta} \in A^{\delta}(\cdot)$ strategy, defines the first player's $x$ trajectory on $[0,2 \delta T]$ interval. This itself by means of $\beta_{\delta}$ strategy of the second player defines its $y$ trajectory on $t \in[0,3 \delta T]$ interval and so on.

In lower $\Gamma_{\delta}^{L}(\cdot)$ game the first player chooses the strategy $\alpha_{\delta} \in A_{\delta}\left(x_{0}, y_{0}\right)$, but the second player choo-ses the strategy $\beta_{\delta} \in B^{\delta}\left(x_{0}, y_{0}\right)$. Analogically in $\Gamma^{L, \delta}(\cdot)$ game trajectories on $[0, \delta T],[\delta T, 2 \delta T], \ldots$ intervals in $\left(\alpha_{\delta}, \beta^{\delta}\right)$ situation will be constructed step step that we note it the following way

$$
\chi\left(\alpha_{\delta}, \beta^{\delta}\right)=\left(x\left(\alpha_{\delta}, \beta^{\delta}\right), y\left(\alpha_{\delta}, \beta^{\delta}\right)\right) .
$$

We need the following theorem from the control theorem, its proof comes out from integral's basic features.

Let's discuss a controlling system

$$
\dot{x}_{i}=f_{i}\left(t, x_{i}, u_{i}\right), u_{i}(t) \in U_{i} \subset R^{p_{i}}, i \in N=\{1, \ldots, n\},
$$

that satisfies all above mentioned standard conditions.

Lemma 3.1. For every $\tau_{i}>0$ number and for every $i \in N$ will be found such number $\varepsilon_{i}>0$ and control$\operatorname{ling} U_{i}$ set's $\varepsilon_{i}$-net $U_{i}^{\varepsilon_{i}}$, that controlling (20) sistem's any trajectory $x_{i}$ can be approximable by $x_{i}^{\varepsilon_{i}}$ trajec-tory uniformity measure, that will be formulated by piecewise constant $u_{i}^{\varepsilon_{i}}$ controlling on $[0, T]$ interval by meaning from $U_{i}^{\varepsilon_{i}}$ set so that the following condition will by fulfilled

$$
\sup _{t \in[0, T]}\left|x_{i}(t)-x_{i}^{\varepsilon_{i}}(t)\right|_{n_{i}}<\tau_{i}
$$

From this lemma and from upper and lower games definition in follows, that for any of them can be approximated by near finite positional 
$\Gamma^{L, \delta}\left(x_{0}, y_{0}, T, U^{\varepsilon_{1}}, V^{\varepsilon_{2}}\right)$ and $\Gamma_{\delta}^{L}\left(x_{0}, y_{0}, T, U^{\varepsilon_{1}}, V^{\varepsilon_{2}}\right)$ games respectively where $U^{\varepsilon_{1}}, V^{\varepsilon_{2}}$ respectively $\varepsilon_{1}, \varepsilon_{2}$-nets in $U$ and $V$ sets. More exactly we are having the following theorem.

Theorem 3.3. For any $\tau>0$ number can be found such number $\mu>0$, that a lexicographic upper game $\Gamma^{L, \delta}(\cdot)=\Gamma^{L, \delta}\left(x_{0}, y_{0}, T, U, V\right)$ approiximate by lexicog-raphic upper finite positional $\Gamma^{L, \delta}\left(x_{0}, y_{0}, T, U^{\mu}, V^{\mu}\right)$ game till to with in $\tau$ precision.

Note 3.1. In this theorem approximation is explai-ned so, that in $\Gamma^{L, \delta}\left(x_{0}, y_{0}\right)$ game for every $\left(\alpha_{\delta}, \beta^{\delta}\right)$ situa-tion can be found in $\Gamma^{L, \delta}\left(x_{0}, y_{0}, T, U^{\mu}, V^{\mu}\right)$ game such situation $\left(\alpha_{\delta}{ }^{*}, \beta^{\delta^{*}}\right)$, that in these situations the payoff's functions meanings are different by the norm no more than $\tau$ number.

Lexicographic upper game $\Gamma^{L, \delta}(\cdot)$ (or lower game $\left.\Gamma_{\delta}^{L}(\cdot)\right)$ represents finite-step-type by turns perfect informational game. Because of the theorem 3.3 this game by means of payoff's function any degree exactness can be approximated by finite positional lexicographic game, where equilibrium situation in pure strategies will by existed. From here and from lemma 2.1 follows the following theorem's correctness..

Theorem 3.4. Lexixographic upper game $\Gamma^{L, \delta}\left(x_{0}, y_{0}\right)$ (in lower game $\Gamma_{\delta}^{L}\left(x_{0}, y_{0}\right)$ ) for any $\varepsilon>0$ number there exists a lexicographic $\varepsilon$-equilibrium.

Let's discuss one more auxilary discrete lexicog-raphic game with incomplete information and note it the following way $\Gamma^{L}\left(x_{0}, y_{0}, \delta\right)$. In this game the players at every move choose control simultaneously, but they don't have information about this move, about his or her partner's choice, but they know both players choice done at previous move.

In this game as in $\Gamma^{L, \delta}(\cdot)$ game by the couple of strategies $\left(\alpha_{\delta}, \beta_{\delta}\right) \in A_{\delta} \times B_{\delta}$ identically will be constructed the only couple of trajectories, we note it so $(x, y)=\chi\left(\alpha_{\delta}, \beta_{\delta}\right) . \quad \Gamma^{L}\left(x_{0}, y_{0}, \delta\right) \quad$ is given from $\Gamma^{L}\left(x_{0}, y_{0}\right)$ game by means of epimorphism:

$$
\mu_{2}: B^{\delta} \rightarrow B_{\delta}, \mu_{2}=\Pi_{\delta}^{Y} \circ \beta^{\delta} .
$$

Theorem 3.5. For any $\varepsilon>0$ number in lexicographic multistag $\Gamma^{L}\left(x_{0}, y_{0}, \delta\right)$ game there exists a lexicographic $\varepsilon$-equilibrium situation.

Proof. $\Gamma^{L}\left(x_{0}, y_{0}, \delta\right)$ game represents the game with incomplete information, where the players simultaneously choose their "lower" dominated strategies. Epimorphism $\mu_{2}$ connect $\Gamma^{L}\left(x_{0}, y_{0}, \delta\right)=\Gamma^{L}($.) game to perfect informational $\Gamma^{L, \delta}(\cdot)$ game, moreover in image and inverse image payoff function's meanings are diffe-rent by number $\varepsilon(\delta) \underset{\delta \rightarrow 0}{\rightarrow} 0$. As by theorem 3.4 in $\Gamma^{L, \delta}(\cdot)$ game there exists lexicographic $\varepsilon$-equilibrium situation, therefore in $\Gamma^{L}(\cdot)$ game by lemma 2.1 there exists a lexicographic $\varepsilon$-equilibrium situation as well.

Now let's discuss in a normal form the basic lexicographic differential game $\Gamma^{L}\left(x_{0}, y_{0}\right)$. In this game the first player's strategic couple

$$
\alpha=\left(\delta_{1},\left\{\alpha_{\delta}\right\}_{\delta=\frac{1}{2^{n}}}\right),
$$

where $\delta_{1}$ is $[0, T]$ interval's rank of binary partition, but $\left\{\alpha_{\delta}\right\}_{\delta=\frac{1}{2^{n}}}, n=1,2, \ldots$ represents the whole sum of the first player's strategies in every multistage games $\Gamma_{\delta}^{L}(\cdot)$, $\delta=\frac{1}{2^{n}}, n=1,2, \ldots$.

In $\Gamma^{L}\left(x_{0}, y_{0}\right)$ game the second player's strategy is the couple

$$
\beta=\left(\delta_{2},\left\{\beta_{\delta}\right\}_{\delta=\frac{1}{2^{n}}}\right)
$$

where $\delta_{2}$ is $[0, T]$ interval's rank of binary partition, but $\left\{\beta_{\delta}\right\}_{\delta=\frac{1}{2^{n}}}, n=1,2, \ldots$ is the whole sum of the second player's in every multistage games $\Gamma^{L, \delta}(\cdot)$, $\delta=\frac{1}{2^{n}}, n=1,2, \ldots$.

In $(\alpha, \beta) \in A \times B$ situation, where $A$ is the first player's set of every strategies in a game $\Gamma^{L}(\cdot)$, but $B$ is the second player's set of every strategies in the same game, couple of trajectories $(x, y)=\chi(\alpha, \beta)$ will be constructed in the following way: we choose a minimal number from two numbers $\bar{\delta}=\min \left\{\delta_{1}, \delta_{2}\right\}$ and in $\Gamma^{L}\left(x_{0}, y_{0}, \delta\right)$ game we will construct the couple of trajectories in $\left(\alpha_{\bar{\delta}}, \beta_{\bar{\delta}}\right)$ situation.

The process of $\Gamma^{L}(\cdot)$ game takes place in the following way: the players name $\delta_{1}$ and $\delta_{2}$ numbers, choose the game $\Gamma^{L}\left(x_{0}, y_{0}, \delta\right)$ where $\delta=\min \left\{\delta_{1}, \delta_{2}\right\}$, but then play $\Gamma^{L}\left(x_{0}, y_{0}, \delta\right)$ game, where a lexicographic $\varepsilon$ equilibrium situation exists. Each play is guaranteed by the meaning of the game till its $\varepsilon\left(\delta_{i}\right)$ number with exactness. Therefore from the theorem 3.5 follows the following theorem.

Theorem 3.6. In lexicographic differential $\Gamma^{L}\left(x_{0}, y_{0}\right)$ game with perfect information for any $\varepsilon>0$ there exists a lexicographic $\varepsilon$-equilibrium situation in pure strategies. 


\section{CONCLUSION}

In differential antagonistic games in scalar payoffs' situation the existence of the solution and the problem of its finding is complex to decide. The equilibrium situation (saddle point or a $\varepsilon$-saddle points) are discussed in such role of the solution. The problems are studied and will be studied by different authors. About lexicographic differential antagonistic $\Gamma^{L}\left(x_{0}, y_{0}\right)$ game we can say that the problem of its solution's existence is rather $n$ difficult. In perfect information conditions are studied such games, that makes easier relevant procedures. For the purpose to approach the unknown optimal strategies the auxiliary upper and lower games $\Gamma^{L, \delta}(\cdot), \Gamma_{\delta}^{L}(\cdot)$ are constructed for making of sequential moves player's. Sets and strategies $A_{\delta}\left(x_{0}, y_{0}\right), A^{\delta}\left(x_{0}, y_{0}\right), \alpha_{\delta}, \alpha^{\delta}, B_{\delta}\left(x_{0}, y_{0}\right)$, $B^{\delta}\left(x_{0}, y_{0}\right), \quad \beta_{\delta}, \beta^{\delta}$ of discrete type of strategies are constructed having certain features on the intervals $[0, \delta T],[\delta T, 2 \delta T], \ldots$ for the unknown $\varepsilon$-equilibrium situation approximate optimal situations are constructed, with the help of them, that in $\Gamma^{L}\left(x_{0}, y_{0}\right)$ game here exists lexicographic $\varepsilon$-saddle point in pure strategies.

\section{REFERENCES}

[1] M.E.Salukvadze. "Vector-Valued Optimization Problems in Control Theory". Academic Press, New York, 1979, $219 \mathrm{p}$.

[2] I.Flugge-Lotz, H. Marbach. "The Optimal Control of Some Attitude Control Systems for Different Performance Criteria". Journal Basic Engrn., Series D, Vol. 85, No. 2, 1963.

[3] W.L.Nelson. "On the Use of Optimization Theory for Practical Control System Design". IEEE Trans, on Auto. Control, Vol. AC-9, No. 4, 1964.

[4] V.I. Zhukovskiy, M.E. Salukvadze. "The Vector-Valued Maximin". Academic Press, New York. 1993, 312 p.

[5] G. N. Beltadze. "Sets of equilibrium situations in lexicographic noncoalition games". Bulletin of the Acade- my of sciences of the Georgian SSR, 98, № 1,1980, pp. 41-44 (in Russian).

[6] G. N. Beltadze. "A mixed extension of finite noncoacoalition lexicographic games".Bulletin of the Academyof sciences of the Georgian SSR, 98, № 2, 1980, pp. 273-276 (in Russian).

[7] G.N.Beltadze. "Analysis of the infinite dimensional lexicographic games". Bulletin of the Academy of sciences of Georgian, 141, № 2, 1991, pp. 241-244 (in Russian).

[8] G. N. Beltadze, A.L. Topchishvili. "Multicriteria noncooperative games with strictly ordered criteria". A.Gop-fert, J. Seelender, Chr. Tammer (Eds). Methods of Multicriteria Decision Theory, Proceedings of the 6 th Workshop of the DGOR -Working Group Multicriteria Optimization and Decision, Frankfurt, 1997, pp. 69-86.

[9] M. E. Salukvadze, G.N. Beltadze, F. Criado. "Dyadic theoretical games models of decision - making for the lexicographic vector payoffs". International Journal of information Technology and Decision Making, Vol. 8, Issue 2, 2009, pp. 193-216.

[10] G. N. Beltadze. "Lexicographic noncooperative game's mixed extension with criteria". International Journal of
Systems and Sofware ARPN Publishers, Vol 1, № 8, November 2011, pp. 247- 250.

[11] G.N. Beltadze. "Lexicographic Multistage Games with Perfect Information". Informational and Communica-tion technologies - Theory and Practice: Proceedings of the International Scientific Conference ICTMC- 2010 Devoted to the $80^{\text {th }}$ Anniversary of I.V. Prangishvili. Nova Publishers, 664 pp. USA, 2012, pp. 275- 281.

[12] G. N. Beltadze. "Lexicographic Strategic Games' Nonstandard Analisis". International Journal of Intelligent Systems and Applications. Hong Kong, Volume 5, Number 7, 2013, pp. 1-8.

[13] G. N. Beltadze, J. A. Giorgobiani. "Shapley's Axiomatics for Lexicographic Cooperative Games'. Internati-onal Journal of Modern Education and Computer Science (IJMECS). Hong Kong, Volume 7, Number 8, August 2015, pp. 1-8.

[14] G. N. Beltadze, J. A. Giorgobiani. "The Stability of Equilibrium Situation in Lexicographic Strategic Games". International Journal of Modern Education and Computer Science (IJMECS). Hong Kong,Volume 8, Number 8, December 2016, pp. 38-45.

[15] M. E. Salukvadze, G. N.Beltadze. "Stochastic Game with Lexicographic Payoffs". International Journal of Modern Education and Computer Science (IJMECS). Hong Kong, Volume 10, Number 4, April, 2018, pp. 10-17.

[16] L. D. Berkovits. "A variational approach to differential games". In: Dresher M., Shapley L.S., Tucker A.W. (eds). Advances in game theory. Anals of mathematics studies,, $\mathrm{Vol}$ 52, Princeton University Press, Princeton, 1964,pp. 127-174.

[17] W.H.Fleming. "The convergence problem for differential games". Journal of Mathematical Analysis and Applications. 1961, Vol 3, Issue 1, pp. 102-116.

[18] P.P. Varaiya, J. Lin. "Existence of saddle points in differential games". SIAM Journal on Control. 1969, Vol 7, pp. 141-157.

[19] P.P. Varaiya. "On the existence of solutions to a differential game". SIAM Journal on Control. 1967, Vol 5,pp. 153-162.

[20] A. Friedman. "Differential games". New York: WileyInterscience, -Pure and applied mathematics (Interscience Publishers), 1971, p. 350.

[21] O.A. Malafeev. "The existence of equilibria in noncooperative two persons differential games with independent movements". Vestnik LGU, Ser. Mathematics, 1980, Vol 4, pp. 12-16.

[22] G.N.Beltadze. "ع-Equilibrium in Games with Strictly Ranked Criteria". Information and Computer Technology, Modeling and Control. Chapter 38. Editors: Ivane Gorgidze, Tamar Lominadze, Maka Khartishvili and Ketevan Makhashvili (Georgian Technical University, Tbilisi, Georgia).Nova Science Publishers, New York, 2017.

\section{Author's Profile}

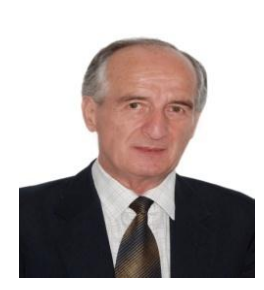

Guram N. Beltadze: is Professoremeritus at Informatics and Control Systems Faculty. He is a mathemati-cian, finished Mathematical Faculty Tbilisi State University. He got a post-graduate education in the Academy of Sciences of the USSR in St.-Petersburg. 1982 - Ph.D. at St.-Petersburg State University, 1992 Dr. Phys.-Math. Sci. at St.-Petersburg State University. He was 
teaching at Tbilisi State University and other Universities. The research area: Game Theory, Operation Research, Decision
Theory, Learning Organization, Mathematical modeling of social economics and political processes.

How to cite this paper: Guram N. Beltadze, " Differential Antagonistic Games with Lexicographic Vector-Payoffs", International Journal of Modern Education and Computer Science(IJMECS), Vol.11, No.3, pp. 23-30, 2019.DOI: 10.5815/ijmecs.2019.03.04 